\title{
Insomnia: what is currently available
}

\author{
A Marais, ${ }^{1}$ E Osuch $^{2}$ \\ ${ }^{1}$ Department of Pharmacology, School of Medicine, Faculty of Health Sciences, University of Pretoria, South Africa \\ ${ }^{2}$ Department of Pharmacology and Therapeutics, School of Medicine, Sefako Makghato Health Sciences University, South Africa \\ Corresponding author, email: dramarais@gmail.com
}

Insomnia is an important public health burden and is the most ubiquitous sleep disorder in the general population, requiring accurate diagnosis and effective treatment. Sleep hygiene and cognitive behavioural therapy form the foundation of management. In addition, various pharmacological entities are at hand to augment insomnia disorder. Acute insomnia requires short-term management with appropriately indicated hypnotic agents, while chronic sleeping difficulties benefit more from antidepressants. This article informs the reader about the currently available sleeping agents in South Africa, and may not include more effective or potent agents used in other parts of the world that are not yet accessible for local prescription.

Keywords: insomnia, antidepressants, antihistamines, benzodiazepines, melatonin, non-benzodiazepines

Traditionally, insomnia is defined as the subjective perception of persistent difficulty in initiating, maintaining and achieving quality sleep which results in associated daytime impairment, notwithstanding sufficient opportunity or external environmental factors to do so. ${ }^{1}$ Epidemiological surveillance studies from the general population illustrate that between $19 \%$ and $44 \%$ of adults experience some form of sleeping difficulty or abnormality, of which $10-15 \%$ of those conform to the official diagnostic criteria for insomnia according to the fifth edition of the Diagnostic and Statistical Manual of Mental Disorders (DSM V). ${ }^{2}$ Incidence rates vary significantly between different countries and communities. The prevalence of insomnia and related symptoms is still poorly understood. However, factors such as socio-demographic conditions, health status and risk behaviour, stress, support systems, education, income, age and gender have all been previously described. ${ }^{3}$ Nocturnal sleep problems seem to be present in approximately $30 \%$ of the South African population, with a slight predisposition towards females (31.3\%) compared to males (27.2\%). ${ }^{4}$

Insomnia disorder is often associated with comorbid psychiatric or medical conditions, including alcohol or substance abuse. For this inference, few studies are available to quantify the exact prevalence of insomnia as being the principal reason for visiting a general practitioner, yet independent observations tend to suggest approximately $10 \%$ of all GP visits are related to sleeping complications being the focus of the consultation. ${ }^{5}$ Although insomnia is the most common sleeping disorder, it is estimated that only $50 \%$ of patients encountering sleeping difficulties consult their family physician or general practitioner for medical assistance or advice. ${ }^{6}$ Insomnia is more prevalent in women and the older population and has been associated with high societal costs and lower quality of life scores, resulting in increased hospitalisations, physician visits and absenteeism. It is estimated that insomnia costs the US economy approximately $\$ 100$ billion annually. ${ }^{7}$
The disorder is classified as either short term (acute) or chronic (long term) depending on the duration of symptoms. Short-term insomnia, also known as adjustment or acute insomnia, usually manifests in response to an identifiable stressor and symptoms do not exceed a period of three months. Chronic insomnia is diagnosed when symptoms occur at a frequency of at least three times per week and have been present for three months or longer.

\section{Consequences of insomnia}

When the normal sleep process is delayed or disrupted, it is natural to harbour feelings of stress, which in turn results in a vicious cycle of insomnia. Recuperative sleep is necessary for general physical health and mental agility. In addition, sleep quality has a direct effect on a person's productivity, emotional well-being, immune status and weight control. Although sleep requirements may vary individually, most healthy adults require at least seven hours of sleep per night to function at their best. ${ }^{8}$ Sleep duration less than seven hours has been associated with a higher incidence of non-communicable chronic diseases such as obesity, hypertension, dyslipidaemia, metabolic syndrome (including diabetes), coronary heart disease, depression, and even colorectal cancer. ${ }^{9}$ Excessive daytime sleepiness as a result of insomnia, sleep loss, sleep fragmentation or shift work is responsible for nearly $20 \%$ of all traffic accidents and sleeprelated fatalities in individuals sleeping less than five hours in a 24 hour cycle. ${ }^{10}$

\section{Overview of pharmacological modalities}

An abundance of pharmacological and non-pharmacological treatment options is available for various types of sleeping disorders. However, sleep hygiene advice and stimulus control form the pillar in managing both short- and long-term insomnia. Cognitive behavioural therapy (CBT) is regarded as the first-line treatment for insomnia. Nonetheless, this requires specialised settings and is not easily available to many patients dependent 
on South Africa's public healthcare system. For this reason pharmacological approaches are mostly used in the clinical setting. ${ }^{11}$ Hypnotics are regarded as having the best short-term efficacy, while antidepressants are considered the best option for managing long-term sleep disorders.

Treatment comparisons regarding sleep onset latency (SOL) (the amount of time it takes from being fully awake to sleep), wakefulness after sleep onset (WASO) (periods of wakefulness occurring after defined sleep onset) and the amount of total time spent sleeping, are often used to decide which hypnotic is best suited for a specific sleeping disorder. A presumably short acting drug with a duration of $<8$ hours is recommended in patients with sleep-onset insomnia or those having difficulty falling asleep (zolpidem or triazolam). Patients with sleep maintenance insomnia, or those waking up in the middle of the night require longer acting drugs such as zolpidem extended release, zopiclone, temazepam, or lorazepam. Table I illustrates the indirect comparisons of the available hypnotic agents in South Africa and represents averages of the oral dosage forms and strengths, since sleep parameters differ significantly between individuals.

Table 1: Indirect comparison of half-life, sleep-onset-latency and total sleeping time of available South African hypnotics

\begin{tabular}{|c|c|c|c|}
\hline Drug & $\begin{array}{c}\mathrm{T} 1 / 2 \\
\text { (hours) }\end{array}$ & $\begin{array}{c}\text { SOL* }^{*} \\
\text { (minutes) }\end{array}$ & $\begin{array}{c}\text { Total sleep } \\
\text { time (hours) }\end{array}$ \\
\hline \multicolumn{4}{|l|}{ Benzodiazepines } \\
\hline \multicolumn{4}{|l|}{ Short acting } \\
\hline Triazolam & $2-3$ & 26.7 & 6.7 \\
\hline Midazolam & $1.5-2.5$ & 25.3 & 7.1 \\
\hline \multicolumn{4}{|l|}{ Intermediate acting } \\
\hline Oxazepam & $10-20$ & 14.9 & 7.4 \\
\hline Lorazepam & $10-20$ & 34.6 & 7.4 \\
\hline Loprazolam & $7-8$ & 37.1 & 7.3 \\
\hline Alprazolam & $12-15$ & 8.6 & 7.5 \\
\hline Temazepam & $10-40$ & 16.7 & 7.7 \\
\hline \multicolumn{4}{|l|}{ Long acting } \\
\hline Flurazepam & $40-100$ & 45.4 & 6.8 \\
\hline Diazepam & $20-80$ & 19.7 & 7.4 \\
\hline Nitrazepam & $35-45$ & 27.2 & 8.1 \\
\hline \multicolumn{4}{|c|}{ Non-benzodiazepines } \\
\hline Zolpidem & 3 & 12.8 & 7.5 \\
\hline Zopiclone & 5 & 11 & 7.1 \\
\hline \multicolumn{4}{|c|}{ Melatonergic agents } \\
\hline Melatonin & $0.5-1$ & 8.3 & 3.5 \\
\hline \multicolumn{4}{|c|}{ Antidepressant drugs } \\
\hline Trazodone & $10-12$ & 26.2 & 6.7 \\
\hline Mirtazapine & $20-40$ & 11.7 & 6.9 \\
\hline Amitriptyline & $20-30$ & 37.7 & 5.9 \\
\hline \multicolumn{4}{|l|}{ Antihistamines } \\
\hline Diphenhydramine & $4-6$ & 34.2 & 6.6 \\
\hline Doxylamine & $10-15$ & 52.5 & $\mathrm{~N} / \mathrm{A}$ \\
\hline
\end{tabular}

\section{Pharmacological strategies}

Any approved pharmacological class of sedatives and hypnotics is superior to placebo in improving short-term sleep outcomes and effectivity. However, the potential benefits of pharmacotherapy remain debatable due to post-marketing pharmacovigilance reports, overestimation of risk/benefit ratio, addiction potential, sleep quality and daytime function to mention a few. ${ }^{1}$ Although various drug groups are employed worldwide, only the currently available agents on the South African market indicated for sleeping disorders will be further discussed.

\section{Benzodiazepines}

Benzodiazepines have been available for treating insomnia since the mid 1950s. These drugs act as agonists at $\gamma$-aminobutyric acid $\left(G A B A_{A}\right) \alpha 1, \beta$ and $\gamma$ neuroreceptors and have far less overdose danger and abuse potential than barbiturates and opioids used for sleep. ${ }^{12}$ Benzodiazepines are beneficial in increasing the sleeping time and improving the quality of sleep by reducing the sleep onset latency and wakefulness after sleep onset. ${ }^{13}$ Most benzodiazepines are extremely effective in inducing sleep, but are frequently associated with ataxia, sedation, daytime drowsiness and cognitive impairment. ${ }^{14}$ All benzodiazepines can result in respiratory depression, especially in patients with pulmonary disease. Tolerance and addiction develop quickly with continuous use. Agents such as flurazepam and diazepam have active metabolites with extraordinarily long half-lives which can last up to 11 days. This makes them unfavourable to prescribe in elderly patients as falls with hip fractures can result. Recently it has been shown that taking benzodiazepines with half-lives exceeding 20 hours, or chronic administration for periods longer than three years have a significantly increased risk for developing Alzheimer's disease. ${ }^{15}$ Withdrawal from longacting agents can be difficult, causing an initial syndrome of insomnia followed by persistent anxiety, depression, nausea and perceptual changes (hallucinations) extending beyond the halflife of the agent for up to three weeks. Withdrawal from shortacting agents has similar symptoms but may develop within a few hours after discontinuation. ${ }^{16}$

\section{Non-benzodiazepine hypnotics}

Non-benzodiazepine drugs, also known as "Z" drugs (zolpidem and zopiclone) are chemically dissimilar to the traditional benzodiazepine agents. They are still referred to as "newer" sedative-hypnotics, although some of them (zolpidem) have been available for almost 26 years. ${ }^{17}$ These agents slow brain activity by selectively binding to the a1 subunit of the GAGA receptor which is primarily responsible for the sedative/hypnotic activity of benzodiazepine drugs. ${ }^{18}$ Their lack of binding to other $\mathrm{GABA}_{\mathrm{A}}$ subunits ( $\beta$ and $\gamma$ ) make them a popular choice in treating adults who have difficulty in falling asleep or staying asleep. Unlike traditional benzodiazepines, the $Z$ drugs have minimal impact on sleeping stages and do not display REM sleep rebound. In addition, their receptor selectivity ensures a more favourable side-effect profile regarding the development of tolerance and the potential for dependence. $Z$ drugs are metabolised by the liver and the dosage needs to be adjusted in 
hepatic dysfunction or the elderly. None of these agents should be used during pregnancy or lactation.

Zolpidem and zopiclone may cause impaired memory and psychomotor retardation. Recent reports on the safety of nonbenzodiazepine drugs have resulted in updated black box warnings cautioning against sleepwalking, sleep driving and engaging in any other activities while not fully awake after taking these medicines. Patients with a prior history or those experiencing any complex adverse sleep behaviour, even after a single dose, should abstain from future ingestion of the offending agent, although the incidence is very rare. Physicians should initiate treatment with the lowest dose possible for the shortest period of time. ${ }^{19}$

\section{Melatonergic drugs}

Insomnia resulting from disruptions in the circadian rhythm where endogenous melatonin levels do not naturally increase before sleep onset (such as jet lag, shift work or old age) may benefit from melatonin extended-release agents. Melatonin is beneficial in increasing the total sleeping time and decreasing onset of sleep in patients older than 55 years, children suffering from autism spectrum disorder or attention-deficit/ hyperactivity disorder, patients with depressive disorders and females with premenstrual dysphoric disorder. ${ }^{20}$ These drugs act as chronobiotics and are capable of shifting the phase of the circadian timing system to mimic the natural endogenous melatonin release required to activate pathways responsible for sleep initiation. ${ }^{21}$ Until recently, melatonin was available as an over-the-counter agent, but has been re-scheduled to schedule four, necessitating a prescription. Side-effects are uncommon but may include blood abnormalities (leukopenia, thrombocytopenia), CNS side-effects, visual disturbances and drug interactions by inducing CYP3A hepatic isoenzymes. ${ }^{22}$

\section{Antidepressant drugs}

Sedating antidepressants are often used off-label to treat insomnia. For this reason, dosing recommendations and guidelines are unavailable, and the tolerability and safety of these agents are unknown. The most commonly used antidepressants in sleeping disorders are mirtazapine, trazodone and amitriptyline. Subtherapeutic antidepressant doses of trazodone have been reported to cause an improvement in sleep quality, and despite a lack of guidelines, remains the second most frequently prescribed agent (following zolpidem) for insomnia in the USA. ${ }^{23}$

Low doses of tricyclic antidepressants (TCA), such as amitriptyline, are useful in managing chronic pain that may be responsible for sleep disturbances. Insomnia caused by depression will additionally find benefit from TCAs. Similarly, mirtazapine, a noradrenergic and specific serotonergic agent with significant antihistaminergic properties, is beneficial in patients with comorbid depression. In general these antidepressants produce sedation by blocking acetylcholine, noradrenaline and serotonin presynaptic receptors with mainly urinary retention and hypotension as adverse effects. ${ }^{23}$ The use of antidepressants for insomnia could be justified in chronic treatment when long-term use of benzodiazepines would potentially result in tolerance and addiction.

\section{Sedating antihistamines}

Sedating $\mathrm{H}_{1}$ antihistamines (diphenhydramine and doxylamine) are available as over-the-counter medication, but have a negligible effect on sleep quality compared to benzodiazepines. They have a significant affinity for other receptor subtypes, including muscarinic receptors resulting in severe anticholinergic side-effects (dry mouth, blurred vision, constipation and confusion). ${ }^{24}$ Older individuals are more prone to these effects and they are more pronounced with longer acting agents such as doxylamine. Tolerance to the effects of diphenhydramine develop within a week. The most useful off-label clinical utility of antihistamines may be argued in patients with associated allergic symptoms or upper respiratory infections causing insomnia. Currently, antihistamines are not recommended for insomnia due to the lack of demonstrated efficacy and high incidence of adverse effects. ${ }^{1}$

\section{Conclusion}

Insomnia is a common condition experienced by males and females from every age group, but healthcare practitioners often underestimate its prevalence. Physicians need to actively ask patients about insomnia symptoms and encourage good sleep hygiene practices. In the short term, insomniacs can be effectively managed by a combination of pharmacological and non-pharmacological modalities. Pharmacological treatment with benzodiazepines or benzodiazepine-like agents should not exceed two to four weeks. Antihistamines and antidepressants should be used with caution due to their anticholinergic sideeffects. The diagnosis and treatment have a high functional success rate and clinicians need to harbour a greater awareness in the clinical evaluation and management of insomnia. In addition, doctors need to embrace the evidence that non-pharmacological treatment is superior in the long-term management of sleeping disorders and should refrain from prescribing hypnotics for chronic and continuous periods.

\section{References}

1. Sateia MJ, Buysse DJ, Krystal AD, Neubauer DN, Heald JL. Clinical Practice Guideline for the Pharmacologic Treatment of Chronic Insomnia in Adults: An American Academy of Sleep Medicine Clinical Practice Guideline. J Clin Sleep Med. 2017;13(2):307-49. PMCID:Pmc5263087. https://doi.org10.5664/jcsm.6470.

2. Bjorvatn $B$, Meland $E$, Flo $E$, Mildestvedt T. High prevalence of insomnia and hypnotic use in patients visiting their general practitioner. Fam Pract. 2017;34(1):20-4. https://doi.org/10.1093/fampra/cmw107.

3. Cunnington $D$, Junge $M F$, Fernando AT. Insomnia: prevalence, consequences and effective treatment. Med J Aust. 2013;199(8):S36-40.

4. Pengpid S, Peltzer K. Prevalence and social and health correlates of insomnia symptoms among middle- and older-age persons in rural South Africa. Journal of Psychology in Africa. 2018;28(6):472-8. https://doi.org10.1080/14330237.201 8.1539897

5. Sivertsen B, Nordhus IH, Bjorvatn B, Pallesen S. Sleep problems in general practice: a national survey of assessment and treatment routines of general practitioners in Norway. J Sleep Res. 2010;19(1 Pt 1):36-41. https://doi. org10.1111/j.1365-2869.2009.00769.x.

6. Bartlett DJ, Marshall NS, Williams A, Grunstein RR. Predictors of primary medical care consultation for sleep disorders. Sleep Med. 2008;9(8):857-64. https://doi. org10.1016/j.sleep.2007.09.002 
7. Wade AG. The societal costs of insomnia. Neuropsychiatr Dis Treat. 2010;7:1-18. PMCID:Pmc3035597. https://doi.org/10.2147/ndt.s15123.

8. Liu Y, Wheaton AG, Chapman DP, et al. Prevalence of Healthy Sleep Duration among Adults - United States, 2014. MMWR Morb Mortal Wkly Rep. 2016;65(6):137-41. https://doi.org/10.15585/mmwr.mm6506a1.

9. Medic G, Wille M, Hemels ME. Short- and long-term health consequences of sleep disruption. Nat Sci Sleep. 2017;9:151-61. PMCID:PMC5449130. https://doi. org/10.2147/nss.s134864.

10. Shekari Soleimanloo S, White MJ, Garcia-Hansen V, Smith SS. The effects of sleep loss on young drivers' performance: A systematic review. PLoS One. 2017;12(8):e0184002. PMCID:PMC5578645. https://doi.org/10.1371/journal. pone.0184002.

11. Frase $L$, Nissen C. Making sleep easier: pharmacological interventions for insomnia. Expert Opin Pharmacother. 2018;19(13):1465-73. https://doi.org/10.1 080/14656566.2018.1511705.

12. Weaver MF. Prescription sedative misuse and abuse. Yale J Biol Med. 2015;88(3):247-56. PMCID:PMC4553644.

13. Balon R. Benzodiazepines revisited. Psychother Psychosom. 2013;82(6):353-4. https://doi.org/10.1159/000353599.

14. Schroeck JL, Ford J, Conway EL, et al. Review of safety and efficacy of sleep medicines in older adults. Clin Ther. 2016;38(11):2340-72. https://doi. org/10.1016/j.clinthera.2016.09.010.

15. He Q, Chen X, Wu T, Li L, Fei X. Risk of dementia in long-term benzodiazepine users: evidence from a meta-analysis of observational studies. J Clin Neurol. 2019;15(1):9-19. PMCID:PMC6325366. https://doi.org/10.3988/jcn.2019.15.1.9.
16. Soyka M. Treatment of benzodiazepine dependence. N Engl J Med. 2017;376(12):1147-57. https://doi.org/10.1056/NEJMra1611832.

17. Monti JM, Spence DW, Buttoo K, Pandi-Perumal SR. Zolpidem's use for insomnia. Asian J Psychiatr. 2017;25:79-90. https://doi.org/10.1016/j.ajp.2016.10.006.

18. Kardos J. The $G_{A B A}$ receptor channel mediated chloride ion translocation through the plasma membrane: new insights from ${ }^{36} \mathrm{Cl}^{-}$ion flux measurements. Synapse. 1993;13(1):74-93. https://doi.org/:10.1002/syn.890130110.

19. Kesselheim AS, Donneyong $M$, Dal Pan GJ, et al. Changes in prescribing and healthcare resource utilization after FDA Drug Safety Communications involving zolpidem-containing medications. Pharmacoepidemiol Drug Saf. 2017;26(6):712-21. https://doi.org/10.1002/pds.4215.

20. Xie Z, Chen F, Li WA, et al. A review of sleep disorders and melatonin. Neurol Res. 2017;39(6):559-65. https://doi.org/10.1080/01616412.2017.1315864.

21. Bartlett DJ, Biggs SN, Armstrong SM. Circadian rhythm disorders among adolescents: assessment and treatment options. Med J Aust. 2013;199(8):S16-20.

22. Semak I, Korik E, Antonova M, Wortsman J, Slominski A. Metabolism of melatonin by cytochrome P450s in rat liver mitochondria and microsomes. J Pineal Res. 2008;45(4):515-23. https://doi.org/10.1111/j.1600-079X.2008.00630.x.

23. Everitt $H$, Baldwin DS, Stuart B, et al. Antidepressants for insomnia in adults. Cochrane Database Syst Rev. 2018;5:Cd010753. PMCID:Pmc6494576. https://doi. org/10.1002/14651858.CD010753.pub2.

24. Culpepper L, Wingertzahn MA. Over-the-counter agents for the treatment of occasional disturbed sleep or transient insomnia: a systematic review of efficacy and safety. Prim Care Companion CNS Disord. 2015;17(6). PMCID:PMC4805417. https://doi.org/10.4088/PCC.15r01798. 